\title{
El sistema de mérito como garantía de estabilidad y eficacia en las sociedades democráticas avanzadas
}

\author{
Víctor Lapuente Giné \\ University of Gothenburg (Suecia) \\ Victor.lapuente@pol.gu.es
}

\begin{abstract}
Resumen
Este artículo resume las contribuciones recientes, tanto teóricas como empíricas, en ciencias sociales sobre la importancia de la "calidad de gobierno" para el bienestar de los ciudadanos de un país. El artículo analiza dos tipos de factores institucionales que pueden facilitar la "calidad de gobierno" y que han sido explorados sistemáticamente en un elevado número de estudios. Por un lado, los factores políticos y, en particular, cómo se seleccionan a las élites políticas en un país. Por otro, factores burocráticos y, en particular, cómo se selecciona a los empleados públicos en un país. Uno de los resultados más importantes de estos estudios es que disponer de una burocracia meritocrática -entendida como una burocracia no politizada- tiene un efecto positivo y significativo sobre la calidad de gobierno en democracias avanzadas.
\end{abstract}

Palabras clave Meritocracia, calidad de gobierno, corrupción, sistema de carrera, países OCDE.

\section{The merit system as a guarantee of stability and performance in advanced democratic societes}

\begin{abstract}
This paper summarizes recent theoretical and empirical contributions in social sciences on the relevance of "quality of government" for understanding cross-country differences in citizens' well-being. The paper explores two types of institutional factors fostering quality of government that have been systematically explored in a large number of studies. In the first place, political factors, and, in particular, how political elites are selected in a country. In the second place, bureaucratic factors, and, in particular, how public employees are selected in a country. One of the most robust findings in this literature is that meritocratic bureaucracy-understood as a non-politicized bureaucracy- has a positive and significant effect on the quality of government in advanced democracies.
\end{abstract}

Key words

Meritocracy, Quality of government, corruption, career system, OECD countries. 


\section{EL PROBLEMA: LA "CALIDAD DE GOBIERNO" EN DEMOCRACIAS AVANZADAS}

\subsection{Definición de "calidad de gobierno"}

Las últimas dos décadas han sido testimonio de un creciente interés, tanto académico como a nivel de practicantes de la administración pública, por lo que algunos denominan "bueno gobierno", otros "buena gobernanza" y otros "capacidad del Estado". ' La primera pregunta que debemos abordar antes de empezar este estudio sobre el "calidad de gobierno" es ofrecer una definición de trabajo del mismo que nos permita luego resumir los avances (y controversias) sobre qué factores llevan al calidad de gobierno y bajo qué circunstancias podemos transformar esos factores en propuestas concretas que puedan conducir al calidad de gobierno/gobernanza ¿Qué queremos decir con "calidad de gobierno"? ¿No se trata de un concepto demasiado normativo que depende de nuestra percepción subjetiva; por ejemplo, de nuestra orientación ideológica? ¿Podemos encontrar fórmulas más o menos estables y más o menos objetivas para diferencias "buen" de "mal" gobierno?

Siguiendo un número creciente de trabajos a nivel comparado y llevados a cabo desde disciplinas diferentes, este estudio sostiene que podemos elaborar una definición de trabajo de "calidad de gobierno" lo suficientemente extensa teóricamente para que resulte un objeto interesante de estudio científico y a la vez lo suficientemente acotada como para que nos permita a posteriori medir esa capacidad de gobierno, tanto en diferentes países como en diferentes niveles administrativos. "Calidad de gobierno" es un concepto sólido desde tres puntos de vista: empírico, teórico, empírico y normativo.

Desde el punto de vista empírico, numerosos trabajos resaltan la alta correlación existente entre diferentes "resultados" (outputs) de un mismo sistema político, tanto a nivel contemporáneo (Holmberg, Rothstein y Nasiritousi 2009) como a nivel histórico (Tabellini 2008, 2009). Aquellos países que obtienen un resultado alto en control de corrupción (tanto en el Corruption Perception Index de Transparencia Internacional como en el control of Corruption de los indicadores de gobernanza del Banco Mundial) también puntúan alto en calidad de la burocracia (por ejemplo, utilizando el índice Bureaucratic Quality, ya sea en su versión del Banco Mundial como en la de organizaciones privadas como International Country Risk Guide), en eficacia gubernamental (medida en indicadores como Government Effectiveness, también del Banco Mundial), así como en imperio de la ley o en protección de la propiedad privada (es decir, en índices de Rule of Law o de Property Rights Protection). Por el contrario, los países que puntúan bajo suelen hacerlo en la mayoría de indicadores. Y los países intermedios

1 Los términos más utilizados en la literatura internacional para referirse a este concepto son "quality of government", "good governance” y "state capacity" (para un breve introducción a estos con114 ceptos, ver Charron y Lapuente 2010). Por simplicidad, utilizaremos "calidad de gobierno" como traduc- 
ocupan también posiciones intermedias en casi todos los índices. Estadísticamente, estos indicadores se hayan tan altamente correlacionados que, como afirma uno de los economistas más influyentes del momento, Guido Tabellini, tiene sentido hablar de "quality of government" (traducido aquí como "calidad de gobierno") como una característica de los estados o entidades políticas. Así, de la misma manera que posicionamos a los países en función de su renta o del nivel de democracia, podemos también clasificar a los países del mundo (o a diferentes entidades subnacionales o locales dentro de cada país) en un continúo de mayor a menor "calidad de gobierno".

Desde el punto de vista teórico, Rothstein y Teorell (2008) ofrecen una definición de "calidad de gobierno" que caracteriza a todos esos estados (u entidades administrativas) que puntúan alto en los diferentes indicadores referenciados arriba. El "calidad de gobierno" sería aquel gobierno que trata a cualquier agente (ya sean ciudadanos, empresas o entidades sin ánimo de lucro) de forma imparcial; es decir, de acuerdo a lo establecido en las normas legales del país. Se trata de una definición de mínimos, pero que permite entender por qué países con tan dispares sectores públicos, como Singapur o Dinamarca, puntúen siempre en el grupo de países con mejor calidad de gobierno. En Singapur el Estado tiene un papel muy reducido en la gestión de la actividad socio-económica y el gasto público es muy inferior a la media de los países desarrollados. Por el contrario, en países del norte de Europa, como Dinamarca u Holanda, el estado tiene un papel regulador más extenso y, además, el gasto público se sitúa frecuentemente por encima del 50 por ciento del PIB. Sin embargo, si bien Singapur y Dinamarca tienen una diferente "cantidad" de gobierno, los dos países comparten una misma "calidad" de gobierno, en el sentido que sus Estados tratan de forma imparcial -sin discriminaciones por razón de género, etnia, o afiliaciones políticas- a aquellos ciudadanos, empresas y entidades con las que interactúan.

Desde un punto de vista normativo, el concepto de "calidad de gobierno" también nos puede ser de utilidad a la hora de diseñar políticas o reformas institucionales destinadas a mejorar la prestación de los servicios públicos. Por ejemplo, una gran preocupación pública tanto en las democracias avanzadas como en los países emergentes es el control de la corrupción. Existe un consenso creciente en la literatura especializada en que las medidas dirigidas de forma directa a combatir la corrupción, como el establecimiento de agencias anti-corrupción, no están produciendo los efectos esperados (Doig y Riley 1998; Johnston 2005; Persson, Teorell, Rothstein 2010). Muchas organizaciones dedicadas exclusivamente a la lucha contra la corrupción acaban siendo capturadas por las propias redes de corrupción o son incapaces de adaptarse a los cambios operativos que las redes de corrupción llevan a cabo para reorientar sus actividades. Si la corrupción es un equilibrio al que tienden la mayoría de agentes públicos y privados en una sociedad, la puesta en marcha de un mecanismo legal o institucional contra la corrupción en una actividad específica, hará simplemente que ésta se desplaze a otro lugar o actividad.

Como afirma uno de los mayores expertos mundiales en corrupción, Daniel Kaufmann, "uno no lucha contra la corrupción luchando contra la corrupción." La corrup- 
ción no debería verse como una enfermedad que puede combatirse directamente, sino más bien como el síntoma de un problema más profundo: la ausencia de "calidad de gobierno". La manera más efectiva de combatir la corrupción es enmarcar dicha lucha dentro de un esfuerzo más amplio de mejora de la "calidad de gobierno". Como se ha visto anteriormente, aquellos países con mayor puntuación en cualquier indicador de "calidad de gobierno" son también los países que presentan menores niveles de corrupción. Además, las investigaciones sobre lucha contra la corrupción encuentran que los factores más efectivos son los relacionados con la construcción de elementos clave de "calidad de gobierno", como una burocracia Weberiana (Dahlström, Lapuente y Teorell 2009).

\subsection{Por qué estudiar la “calidad de gobierno" es relevante}

Durante muchos años, bajo las ideas dominantes del llamado "consenso de Washington", el Estado se ha concebido, por parte de los economistas académicos y de las instituciones dedicadas al desarrollo, más como un problema a minimizar que como una solución activa a los problemas sociales. Sin embargo, durante los años 90, se produce un cambio significativo en la visión que los líderes académicos y de opinión en cuestiones de desarrollo socio-económico ven al Estado. El cambio se puede situar simbólicamente en 1997, cuando el Banco Mundial dedica su informe anual sobre desarrollo al "Estado en un mundo cambiante" (World Bank 1997). Uno de los economistas actuales más influyentes, Lawrence Summers, admite también un cambio de paradigma en su visión de cómo fomentar el desarrollo humano: "una lección abrumadora que creo que hemos aprendido en los 90 es la importancia trascendente que la calidad de las instituciones y las cuestiones, estrechamente relacionadas, de la eficacia de la administración pública" (citado en Besley 2007: 571).

Un buena administración pública y, en general, lo que hemos definido anteriormente como "calidad de gobierno", ha pasado de ser una cuestión secundaria a convertirse en una cuestión central para explicar el éxito o fracaso de una sociedad en cualquier tipo de dimensión. Un gran número de economistas, por ejemplo, ha desplazado su interés de las variables tradicionales para explicar el desarrollo económico, como el capital físico y humano o los recursos naturales, a cuestiones relacionadas con la "calidad de gobierno". La "calidad de gobierno", ya sea medida de forma directa o a través de alguno de sus componentes (de nuevo, es importante enfatizar que los resultados no varían demasiado, ya que los componentes suelen estar todos íntimamente correlacionados), se ha encontrado en numerosos estudios como clave para explicar el crecimiento económico (Acemoglu, Johnson, and Robinson 2004; Acemoglu, North, Rodrik, y Fukuyama 2008; Clague, Keefer, Knack y Olson 1996; Easterly 2001; Hall y Jones 1999; Knack y Keefer 1995; Mauro 1995; Mo 2001; Rodrik, Subramanian, y Trebbi 2004). No sólo los países con menor calidad de gobierno crecen menos, sino que, además, crecen de forma más desigual y presentan mayores niveles 116 de pobreza (Gupta, Davoodi, and Alonso-Terme 2002). Es decir, la falta de imparcili- 
dad en la actividad del Estado perjudica económicamente a todos, pero especialmente a los más desfavorecidos.

La calidad de gobierno puede tener un efecto más importante que variables consideradas claves por los estudiosos de las tradiciones jurídicas o denominados “economistas legales". De acuerdo a una línea de pensamiento que alcanzó una notable influencia durante la última década del pasado siglo, el hecho de que un país perteneciera a una determinada tradición legal - Civil Law o Common Law - tendría un impacto muy notable sobre el desarrollo socio-econónomico del mismo. En una serie de artículos altamente citados, los economistas La Porta, Lopez-de-Silanes, Shleifer y Vishny $(1997,1998,1999,2008)$ mostraron que, dado el mayor "peso de la mano del Estado" en las actividades económicas propia de los países con tradición de Civil Law (como España, Francia o Italia), la tradición legal del Common Law ofrecía mejores perspectivas para el desarrollo a largo plazo de un país. Sin embargo, estudios recientes apuntan a que, la diferencia legal Comon Law versus Civil Law podría estar escondiendo otros legados históricos, como, por ejemplo, la presencia de calidad de gobierno -o, para ser más exactos, de una burocracia meritocrática- durante la creación del estado moderno durante los siglos XVI-XVIII (Charron, Dahlström y Lapuente 2010).

Otros científicos sociales, como politólogos y sociólogos, han desplazado su atención, a su vez, de factores explicativos de índole cultural, como la llamada "confianza social" o el "capital social" para explicar diferencias entre sociedades (por ejemplo, entre el norte y el sur de Italia, en el famoso estudio de Robert Putnam 1993) hacia factores relacionados con la "calidad de gobierno". Así, existe creciente evidencia empírica señalando que no son las sociedades con mayor "capital social" (por ejemplo, por virtud de compartir unos determinados valores de civismo democrático) las que producen los gobiernos con mayor calidad, sino que, por el contrario, lo que viene primero en la relación de causalidad es tener calidad de gobierno (Rothstein y Eek 2009; Rothstein y Stolle 2003). En otras palabras, si estamos interesados en mejorar el funcionamiento de nuestras instituciones democráticas y apuntalar los valores de civismo democrático, el primer lugar en el que operar debería ser intentar construir calidad de gobierno como, por ejemplo, a través de la consolidación de una burocracia meritocrática.

Múltiples estudios en otras disciplinas, desde psicología y medicina hasta ciencias medioambientales o estudios de la paz, muestran los efectos que, en cualquier dimension relacionada con el bienestar de una comunidad tiene el hecho de disponer de un estado imparcial. Tener calidad de gobierno en una sociedad aumenta la esperanza de vida, el nivel educativo y el desarrollo humano en general (Akçay 2006), pero también la percepción subjetiva de felicidad (Frey y Stutzer 2000; Rothstein 2010), la percepción de legitimidad del sistema democrático (Holmberg, Rothstein y Nasiritousi 2009), la protección del medioambiente (Damania 2003, Welsch 2004, Holmberg, Rothstein y Nasiritousi 2009) y, además, reduce significativamente la probabilidad de conflicto civil violento (Fearon and Laitin 2003, Öberg y Melander 2010). 
Es fácil imaginar que los problemas de calidad de gobierno afectan fundamentalmente a los países en vías de desarrollo o emergentes y, por ende, no deberían ser una preocupación pública en democracias avanzadas como las europeas. Sin embargo, si observamos la evolución histórica de los países europeos en cualquier indicadores reconocido de calidad de gobierno, como los mencionados anteriormente procedentes del Banco Mundial, del International Country Risk Guide o de Transparencia Internacional (posiblemente, los tres indicadores más utilizados en los estudios comparados y con los que podemos ver la evolución de los países durante las últimas dos décadas), vemos cómo las teóricamente democracias avanzadas europeas muestran un comportamiento muy diferente. Por una parte, la calidad de la administración pública y el control de la corrupción encuentran su máxima expresión en algunos países europeos, como Dinamarca, Suecia, Finlandia u Holanda.

Sin embargo, muchos países europeos del arco mediterráneo, y en particular, España, Portugal y Francia han perdido posiciones relativas, siendo superados en ocasiones por países no europeos que han avanzado notablemente en control de la corrupción y calidad burocrática, como Chile. Otros países, como Italia o Grecia presentan caídas mucho más notables, cayendo fuera del grupo de 60 o incluso 70 mejores países en algunos indicadores, y, por tanto, con niveles inferiores a países tan diversos como Botswana, Costa Rica, Jordania o Cabo Verde. Más preocupante todavía es la evolución de muchos nuevos miembros de la Unión Europea que, a pesar del acceso al mercado común, presentan niveles de calidad de gobierno que no sólo son bajos, sino que no parecen estar aumentando. Los casos más notorios sería Rumania y Bulgaria, pero países como Polonia, Eslovaquía o Letonia presentan puntuciones por lo general muy bajas. Estos simples trazos sobre la dispar evolución europea en los últimos años en materia de calidad de gobierno nos recuerdan que "si alguien pensaba que los retos de corrupción y buen gobierno eran un monopolio exclusivo de los países en vías de desarrollo... esa noción ha sido descartada por completo (Kaufmann, citado en Rothstein 2009:2). La calidad de gobierno debería, por tanto, ser una preocupación relevante tanto para académicos como para dirigentes públicos y líderes de opinión en sociedades democráticas avanzadas como la nuestra.

\section{POR QUÉ UNOS PAÍSES (O ADMINISTRACIONES) TIENEN MAYOR O MENOR “CALIDAD DE GOBIERNO"}

La creciente evidencia sobre la importancia de la "calidad de gobierno" ha llevado aparejado el surgimiento de una literatura, que empieza ya a ser notable, sobre por qué unos países han sido capaces de consolidar niveles más altos de "calidad de gobierno" que otros. Para una introducción a los avances de esa literatura, los trabajos de Daniel Treisman $(2000,2007)$ resultan altamente recomendables. En esta sección nos centraremos en los factores institucionales, en primer lugar por la naturaleza de esta revista y, en segundo lugar, porque se trata de factores que permiten una

118 mayor acción humana. A diferencia de los valores culturales o religiosos, las institu- 
ciones públicas pueden modificarse y pueden convertirse, de manera relativamente sencilla, en la base de recomendaciones políticas.

La sección está dividida en dos partes. En la primera discutiremos las instituciones públicas inicialmente más analizadas en los estudios comparados: las instituciones políticas. Es decir, los mecanismos que existen en un país para elegir a la clase política dirigente del Estado. En la segunda, analizaremos el paper de las instituciones burocráticas y, en particular, de los sistemas de personal. Es decir, los mecanismos existentes en un país para seleccionar no a las personas que forman la élite política del Estado -tanto en el legislativo como en el ejecutivo- si no la forma de seleccionar, promocionar e incentivar a los funcionarios o empleados públicos. Estos mecanismos relacionados con la gestión de los recursos humanos en la función pública han sido menos explorados tradicionalmente por la literatura, pero una serie de trabajos recientes destaca su papel fundamental a la hora de mejorar la calidad de gobierno en un país.

\subsection{Las instituciones políticas}

Hay una extensa literatura analizando el posible impacto de instituciones que podríamos denominar de forma genérica como "políticas", pues están relacionadas con la cúspide política del aparato del Estado, sobre calidad de gobierno en general o sobre aspectos concretos de la misma (como corrupción). Podemos dividir los factores políticos en cuatro grandes grupos de explicaciones.

En primer lugar, para muchos autores, lo que importa es el tipo de sistema político del país. En principio, cabría esperar que, cuanto mayor sea el nivel de democracia de un país menor, mayor sea la "calidad de su gobierno". En regímenes dictatoriales, semi-autoritarios o "híbridos", los responsables políticos no son elegidos por los ciudadanos y, por consiguiente, deberíamos esperar un menor nivel de responsabilidad a la hora de prestar políticas públicas y una mayor parcialidad. Sin embargo, empíricamente, no aparece de forma tan nítida una relación lineal entre el grado de democracia de un país (medida habitualmente a través de indicadores sobre la limpieza y competitividad electoral) e indicadores de calidad de gobierno.

Por ejemplo, algunos autores, como Harris-White y White (1996: 3) o Sung (2004: 179), consideran que existe una relación "contradictoria" entre democracia y uno de los factores claves de calidad de gobierno: corrupción. Es contradictoria porque se trata de una relación estadísticamente significativa -es decir, parece que hay una relación entre el índice de democracia y la ausencia de corrupción-, pero no se trata de una relación directa lineal, como suele ocurrir con otras relaciones en ciencias sociales. En comparación con los regímenes más autoritarios, las democracias tienen un efecto positivo sobre el control de la corrupción. Pero, durante los primeros años (que en algunos casos, como muchos países ex-comunistas o de América Latina, representan un largo periodo de tiempo), la democracia no mejora, sino que puede in- 
cluso empeorar la lucha contra la corrupción. Procesos de democratización han conllevado descensos en la calidad de gobierno en algunos países, como han mostrado un gran número de estudios de caso (Lemarchand 1972, Scott 1972, Wade 1985). En consecuencia, la literatura comparada considera que la relación entre democracia y corrupción es curvilinear en forma de "U" (para Montinola y Jackman 2002), de “J" (para Bäck y Hadenius 2008) o de "S" (para Sung 2004).

Algo similar ocurre cuando tenemos en cuenta no sólo corrupción sino también otros indicadores de calidad de gobierno. En dos de los estudios recientes más citados, el economista del Banco Mundial Philip Keefer (2007, y con Vlaicu 2008) demuestra que cuánto más vieja es una democracia (medida en función de los años transcurridos desde la última transición exitosa a la democracia), mayor es la calidad de gobierno en un país (medida a través de varios indicadores capturando hasta qué punto las políticas públicas benefician a grupos concretos o, por el contrario, proporcionan bienes públicos que benefician al conjunto de la sociedad). De forma parecida, otros trabajos recientes muestran que, si bien la democracia puede ser un elemento necesario para alcanzar un nivel elevado de calidad de gobierno, la democracia por sí sólo no es un factor suficiente (Welzel y Inglehart 2008; Charron y Lapuente 2010).

El segundo grupo de factores políticos que podrían afectar a la calidad de gobierno hace referencia los sitemas electorales. Existe un debate académico intenso sobre qué sistema electoral es mejor para aumentar la calidad de gobierno y, en especial, para controlar los niveles de corrupción. No existe una respuesta absoluta en el sentido de que uno de los dos sistemas alternativos -el sistema mayoritario o "first-past-the-post" versus el sistema proporcional- resulte claramente superior al otro en términos de calidad de gobierno. Pero, de forma similar a lo que comentábamos en relación a la democracia, que no exista una relación lineal aparente no quiere decir que no exista una relación. Los estudiosos han encontrado que dos características -una propia de cada sistema electoral- producen efectos positivos en la lucha contra la corrupción (Persson, Tabellini and Trebbi 2003; Chang and Golden 2006).

Por una parte, una característica buena de los sistemas electorales mayoritarios es que se vota a candidatos individuales ( $y$ no a listas de partido cerradas) lo que aumenta los incentivos a comportarse honestamente. Ello podría explicar por qué los países anglosajones, en especial Nueva Zelanda, Australia, Canada y Reino Unido, presentan niveles de calidad de gobierno significativamente altos y un número de escándalos de corrupción inferior al de muchos de los países de su entorno. Si los votantes pueden echar a un candidato directamente, como allí sucede, el candidato tendrá un incentivo especial para mantener su reputación intacta. Por otra parte, en muchos sistemas proporcianales (por ejemplo, en la Europa del norte; no en España) la falta de responsabilidad individual se compensa con un mecanismo para corregir que los partidos dominantes entren en una espiral de escánda120 los de corrupción. El mecanismo consiste en el diseño de circunscripciones 
electorales que elijan simultáneamente a muchos representantes. Es decir, en esos países hay pocas "barreras de entrada" para que una opción política nueva pueda entrar en la vida política, pues incluso un modesto porcentaje de voto te garantiza representación.

Es importante señalar que España carece de ambas características, pues, por una parte, no votamos candidatos individuales $\mathrm{y}$, por otra parte, existen numerosas las circunscripciones electorales en las que se eligen a muy pocos representantes. No sólo eso, sino que existen incentivos de todo tipo para favorecer el bipartidismo político y la penalización de facto de las opciones minoritarias (sobre todo, de ámbito estatal). Se puede afirmar por tanto que, a la hora de combatir la corrupción, en materia electoral, tenemos lo peor de cada sistema. Por un lado, carecemos de candidatos a los que podemos premiar o castigar de forma individualmente, de forma contraria a como sucede en los sistemas mayoritarios anglosajones. Por otra parte, tenemos muchas barreras a la hora de votar hacia opciones políticas minoritarias, con lo que no disponemos del mecanismo de control del que sí disfrutan otros ciudadanos europeos con un sistema electoral proporcional.

Un tercer grupo de factores políticos señalados como claves para aumentar la calidad de gobierno en un país no hacen referencia tanto a cómo seleccionamos a las élites políticas, sino a quiénes componen dichas élites. No es el tipo de sistema político, sino el tipo de político lo relevante. En particular, uno de los hallazgos empíricos más sólidos de los últimos años es que, ceteris paribus -es decir, controlando por un largo número de factores, incluido el nivel educativo y el desarrollo económicocuánta mayor es la proporción de mujeres en posiciones políticas de poder del Estado (por lo general, la mayoría de indicadores se centran en el porcentaje de mujeres en los parlamentos nacionales), menor es el nivel de corrupción en un país (Dollar et al.. 2001, Sung 2003). Un problema con este resultado -que, es importante enfatizar, se mantiene constante independientemente del contexto social, económico o cultural de los países analizados- es que no disponemos de un mecanismo teórico consistente sobre por qué un mayor número de mujeres lleva a una mayor calidad de gobierno. Existen diversas hipótesis que son pormenorizadas y criticadas de manera detallada y rigurosa en un reciente estudio de Lena Wängnerud (2008). Muchos interrogantes quedan sin contestar: ¿Se debe las diferencias de género a factores biológicos y, de forma similar a cómo las mujeres comiten un número significativamente menor de crímenes, las mujeres son simplemente menos corruptas que los hombres? ¿O son motivos sociales los que subyacen a esa diferencia; por ejemplo, que las redes de corupción pertenecen a redes sociales más amplias, como los llamados "old boy clubs", donde se socializa desde temprana edad a los hijos varones, pero de los que quedan excluidas las mujeres?

Algunos autores destacan un cuarto grupo de factores políticos que pueden explicar las diferencias entre países en términos de calidad de gobierno. Lo que importaría no sería el tipo de poder político ni el tipo de político, sino la fragmentación del poder político. Comparando diversas democracias, Andrews y Montinola (2004) 
encuentran que aquellos gobiernos en los que existe un mayor número de actores con la capacidad de vetar una política ( $y$, por ende, de vetar una ayuda parcial a un determinado grupo de interés), menor es el nivel de corrupción en un país. Utilizando un argumento parecido, Persson, Roland y Tabellini (2000) sostienen que en los regímenes presidenciales, dado que actores políticos con distintas preferencias (por ejemplo, un congreso republicano y un presidente demócrata en los EEUU) deben ponerse de acuerdo, las posibilidades de llevar a cabo tratos de favor a determinados grupos son más limitadas que en sistemas parlamentarios.

Sin embargo, controlando por otros factores, el argumento sobre la bondad de los sistemas presidenciales no parece tener un apoyo empírico robusto. A nivel más general, podemos concluir que los estudios sobre instituciones políticas si bien son capaces de explicar una parte de la variación entre países en términos de calidad de gobierno, dejan una gran parte de las diferencias sin explicar. Èse es el motivo principal que ha impulsado a una serie de investigadores a desplazar su atención de las instituciones políticas hacia las instituciones burocráticas. Como indicara Johan Olsen (2005) en el título de uno de los artículos pioneros del renacimiento de la burocracia Weberiana como sujeto de estudio en los análisis comparados de calidad de gobierno, "Quizás es tiempo de redescubrir la burocracia".

\subsection{Las instituciones burocráticas}

Los trabajos de Johan Olsen $(2005,2008)$ representan posiblemente las contribuciones teóricas más destacadas en relación al papel que la burocracia Weberiana o meritocrática puede desempeñar en la actualidad para garantizar una prestación de servicios públicos imparciales y exentos de corrupción. En todo caso, como señala Olsen, si bien es posible encontrar una relación entre una burocracia Weberiana y, por ejemplo, el control de la corrupción, a nivel analítico ( $y$, sobre todo a nivel normativo; es decir, si queremos extraer recomendaciones políticas tanto para democracias avanzadas como para países emergentes), recurrir a la burocracia Weberiana como panacea resulta complicado.

En primer lugar, porque no sabemos cuáles de las múltiples características apuntadas por Max Weber (1978) realmente importan para aumentar la calidad de gobierno: ¿Es el hecho de seguir unos procedimientos estandarizados y por escrito lo que importa? ¿o es el sentido de jerarquía? ¿O es el sistema de carrera cerrada asociado tradicionalmente con la burocracia meritocrática? Ademés, un problema añadido es que las características de una burocracia Weberiana no van siempre de la mano. Por ejemplo, hay países muy Weberianos en algunos aspectos, pero que, sin embargo, no disponen de sistemas de carrera cerrada, como Holanda o los países nórdicos.

La mejor manera de acercarse a una realidad tan compleja como la de las buro-

122 cracias Weberianas y su impacto sobre la calidad de gobierno es mediante ejerci- 
cios empíricos lo más exhaustivos posibles. Obviamente, “medir” caractarísticas burocráticas es siempre complicado. Sin embargo, siempre es mejor tener un indicador, por imperfecto que sea, que no disponer de medida alguna. En particular, comentaremos aquí dos ejercicios empíricos basados en entrevistas a expertos en administración pública -Evans y Rauch (1999) y Dahlström, Lapuente y Teorell (2009)- que intentan analizar los componentes de una burocracia Weberiana que realmente importan a la hora de controlar la corrupción (o aumentar la calidad de gobierno).

El hallazgo más destacado en el trabajo pionero de Evans y Rauch (1999) es que la forma en la que se opera en una administración (por ejemplo, más o menos procedimientos formales, más o menos jerarquía) no importa tanto como la gestión de los recursos humanos. El elemento clave de una burocracia Weberiana a la hora de atajar la corrupción y garantizar calidad de gobierno es la existencia de "meritocracia" en el empleo público. A pesar de la notable contribución del estudio de Evans y Rauch (1999), existen diversos límites a las conclusiones que se puede sacar del mismo. En primer lugar, Evans y Rauch analizan 35 países considerados emergentes en los 80 (como España, Corea, Taiwan) y, por tanto, no sabemos si sus resultados se puede aplicar también a sociedades democráticas contemporáneas. En segundo lugar, los 35 países de la muestra contienen un elevado número de los Ilamados "tigres asiáticos" o "dictaduras desarrollistas"; es decir, de países que despegaron económicamente en los años 60 y 70 en un contexto en el que las burocracias centrales jugaron un papel histórico sin muchos precendentes anteriores y también con escasos ejemplos actuales. En otras palabras, es posible que los resultados positivos de las burocracias Weberianas en el estudio de Evans y Rauch (1999) esté sesgado porque fundamentalmente tienen en cuenta ejemplos de burocracias exitosas. Además, el trabajo de Evans y Rauch no deja claro qué características particulares de una burocracia Weberiana meritocrática importan y a través de qué mecanismos dichas características reducen la corrupción en un país.

Para responder a estas cuestiones, Dahlström, Lapuente y Teorell (2009) realizan un estudio en el que participan más de 500 expertos de 52 países, fundamentalmente del entorno OECD y de la Europa del este. Se trata pues del estudio cuantitativo comparado existente más ambicioso, tanto en el número de países analizados como en el número de características burocráticas examinadas. Los expertos, por lo general con conocimientos en administración comparada y que, por tanto, son capaces de situar las administraciones de su país en comparación con las de otro, fueron preguntados sobre diferentes características individuales de las administraciones públicas de sus países. En otras palabras, se les pregunta hasta qué punto en su país existen las caractarísticas representadas en la columna 3 de la tabla 1. 
TABLA 1

Explicaciones alternativas sobre cómo la burocracia Weberiana puede afectar al nivel de calidad de gobierno

\begin{tabular}{|c|c|c|}
\hline $\begin{array}{l}\text { Teoría burocrática sobre } \\
\text { calidad de gobierno }\end{array}$ & $\begin{array}{l}\text { Mecanismos causales } \\
\text { Causal Mechanisms }\end{array}$ & Características observables \\
\hline $\begin{array}{l}\text { 1. Una burocracia cerrada (ej. } \\
\text { un sistema de carrera } \\
\text { cerrada) garantiza el buen } \\
\text { gobierno y el control de la } \\
\text { corrupción }\end{array}$ & $\begin{array}{l}\text { "Esprit de Corps" o } \\
\text { "socialización" }\end{array}$ & $\begin{array}{l}\text { 1. Hasta qué punto existen } \\
\text { exámenes formales de } \\
\text { acceso a la function pública } \\
\text { (ej. oposiciones) } \\
\text { 2. Hasta qué punto el puesto } \\
\text { de trabajo público está } \\
\text { asegurado de por vida } \\
\text { 3. Existen leyes laborales } \\
\text { especiales para los } \\
\text { funcionarios públicos } \\
\text { diferentes de las leyes } \\
\text { laborales para los } \\
\text { empleados del sector } \\
\text { privado }\end{array}$ \\
\hline $\begin{array}{l}\text { 2. Una burocracia } \\
\text { profesional (ej. donde quizás } \\
\text { se selecciona como en el } \\
\text { sector privado, mediante } \\
\text { entrevistas y análisis del CV, } \\
\text { pero de forma despolitizada) } \\
\text { garantiza el buen gobierno y } \\
\text { el control de la corrupción }\end{array}$ & $\begin{array}{l}\text { "Separación de intereses" } \\
\text { entre políticos que son } \\
\text { responsables al partido y } \\
\text { empleados públicos que son } \\
\text { responsables al grupo } \\
\text { profesional al que } \\
\text { pertenecen }\end{array}$ & $\begin{array}{l}\text { 4. Hasta qué punto los } \\
\text { méritos cuentan para } \\
\text { acceder a un empleo público } \\
5 \text {. Hasta qué punto } \\
\text { conseguir un empleo público } \\
\text { depende de conexiones } \\
\text { políticas } \\
\text { 6. Hasta qué punto las } \\
\text { promociones en la } \\
\text { administración pública son } \\
\text { internas }\end{array}$ \\
\hline
\end{tabular}

De esta forma, Dahlström, Lapuente y Teorell contrastan empíricamente tanto la explicación más habitual de por qué la burocracia Weberiana es buena para controlar la corrupción -la idea de una "burocracia cerrada" (teoría 1 en la tabla)- como una hipótesis alternativa que denominan "burocracia profesional” (teoría 2). De acuerdo con la interpretación habitual, una burocracia que dispone de las tres primeras características observables (examenes formales u oposiciones, inamovilidad en el puesto de trabajo, y leyes laborales especiales para los funcionarios públicos) reduciría la corrupción mediante un mecanismo de socialización interna. Tal y como enfatizan Evans y Rauch (1999), las burocracias meritocráticas desarrollan un esprit de corps basado en unos valores compartidos de integridad moral y respecto a la imparcialidad. Con los datos obtenidos en el estudio, Dahlström, Lapuente y Teorell testan esta hipótesis construyendo el indicador de "sistema de carrera cerrada" que vemos en el 124 gráfico 1 y que resume las tres primeras características de la Tabla 1. En el mismo po- 
como positivas para el control de la corrupción, no parece existir relación significativa entre una burocracia cerrada y la habilidad que un país tiene para controlar la corrupción (medida con el indicador del Banco Mundial).

\section{GRÁFICO 1}

"Burocracia cerrada" y control de la corrupción

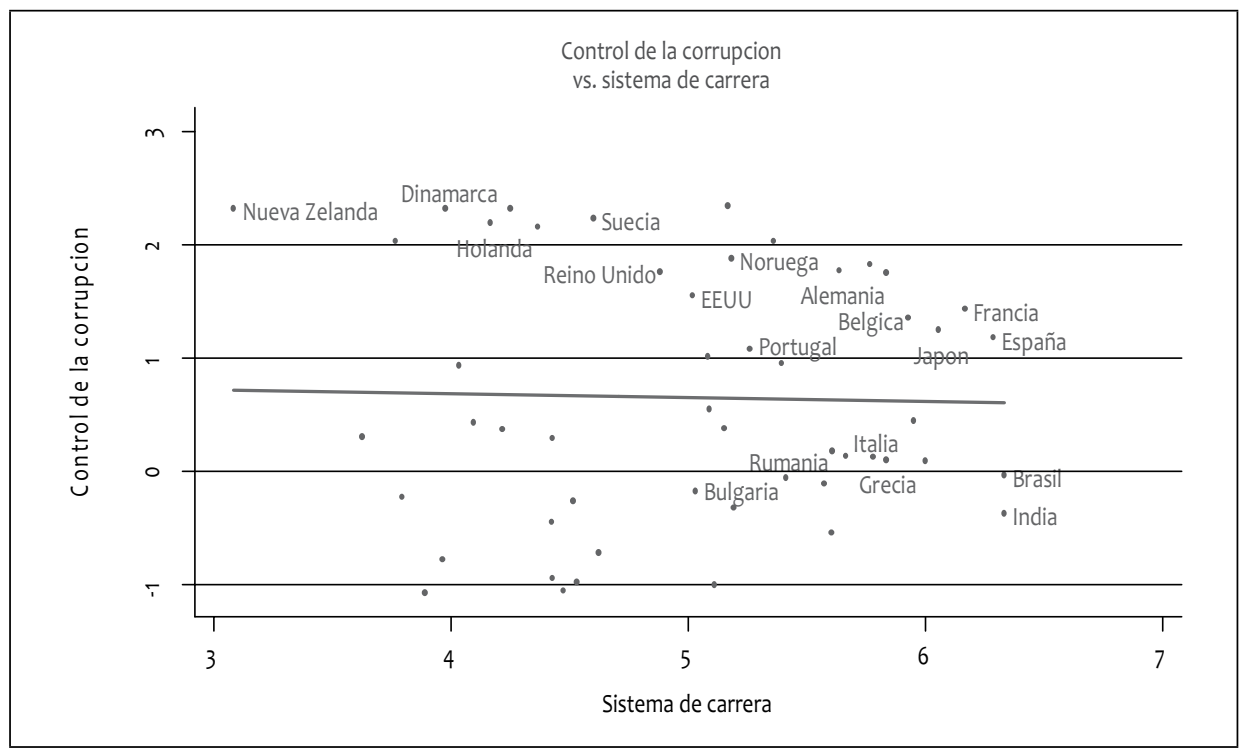

La segunda teoría que Dahlström, Lapuente y Teorell (2009) contrastan empíricamente, y para la que encuentran un apoyo robusto, hace referencia a lo que denominan separación de intereses dentro de la administración. Como los frecuentes ejemplos de corrupción en países con burocracias altamente cerradas y aisladas como Japón demuestran, no es posible inferir automáticamente que los funcionarios sean siempre socializados en los valores adecuados. La hipótesis que estos autores presentan (y que debe entenderse como complementaria y no simplemente como alternativa a la anterior) se basa en los trabajos de economía política de Gary Miller (con Hammond 1994 y con Falaschetti 2001). Dado que el poder público conlleva la posibilidad de abuso siempre, la única manera factible de minimizar el riesgo de abuso consiste en forzar a agentes con intereses distintos a tomar decisiones de forma conjunta. Eso es lo que ocurre en aquellas administraciones donde las carreras (o los intereses) de políticos y empleados públicos están claramente separados. Ello quiere decir que la politizacion "desde arriba” es muy limitada; es decir, que los políticos no controlan el acceso al empleo público ni las promociones internas. Y ello también quiere decir que hay poca politización "desde abajo"; es decir, que los funcionarios públicos tiene límites formales e informales a la hora de pasar a la arena político. El gráfico 2 recoje el efecto que las características 4-6 de la tabla 1 tienen sobre el control de la corrupción en un país. 
GRÁFICO 2

"Burocracia profesional" y control de la corrupción

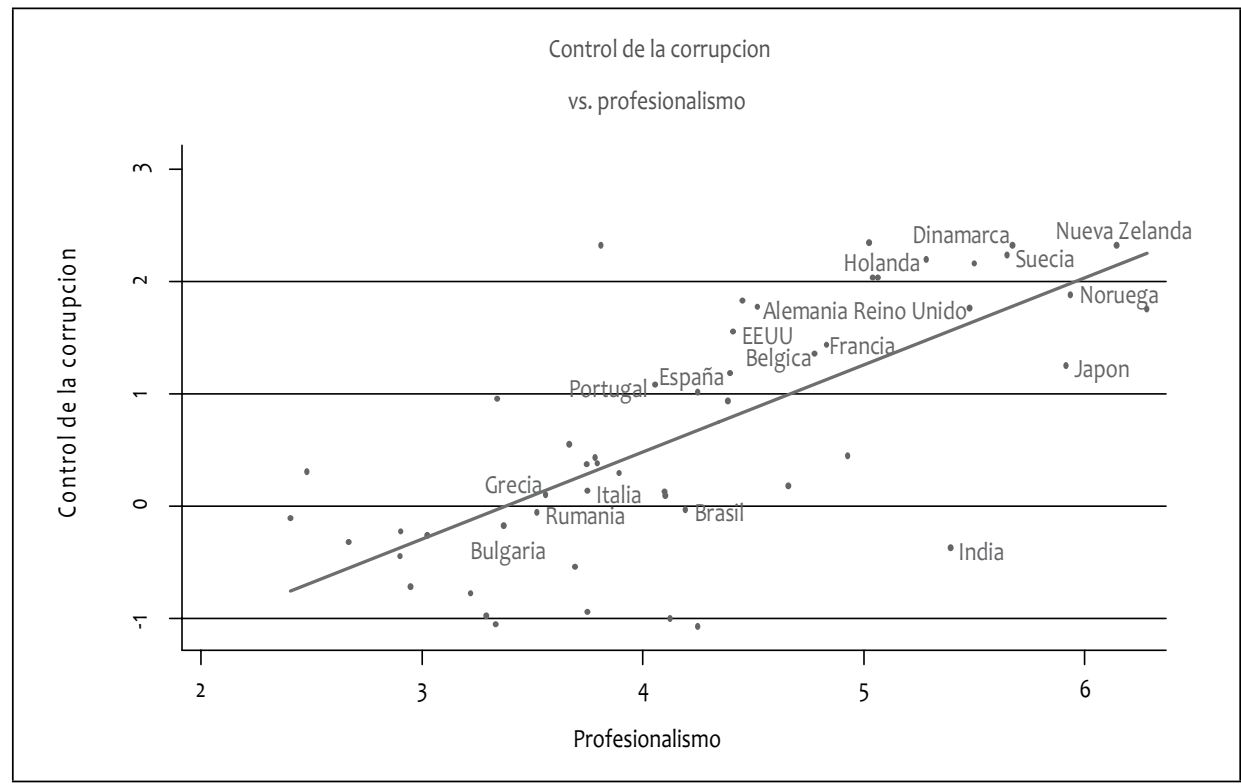

Como podemos ver, el efecto del grado de "profesionalismo" de una burocracia -que fundamentalmente es un agregado de las tres características ligadas a la segunda teoría de la tabla 2- resulta sustancial. Los países con burocracias más profesionales, como Nueva Zelanda, Noruega o Suecia, donde los empleados públicos, a pesar de carecer de oposiciones y de un régimen laboral especial, son reclutados de forma meritocrática, presentan niveles de corrupción significativamente menores que los países con administraciones menos profesionalizadas o, lo que es lo mismo en este caso, más politizadas.

\section{CONCLUSIONES}

Este artículo ha resumido los avances tanto teóricos como empíricos en diversas disciplinas -sobre todo, ciencia política, economía y sociología- sobre la importancia de la calidad de gobierno para el bienestar de los ciudadanos de un país y los posibles mecanismos institucionales que nos pueden ayudar a mejorarla. Muchos interrogantes quedan abiertos, pero dos ideas parece gozar de un creciente consenso y aceptación entre los expertos en calidad de gobierno anivel comparado. En primer lugar, que la calidad de gobierno no es tanto, o no sólo, el resultado de una sociedad "virtuosa" (económicamente o como resultado de tener unos valores culturales concretos), sino también una causa clave para tener una sociedad virtuosa. En otras pala-

126 bras, vale la pena invertir recursos económicos y de investigación en cuestiones 
relacionadas con la calidad de gobierno. En segundo lugar, que, de entre los muchos factores que favorecer la calidad de gobierno, la existencia de una burocracia meritocrática -entendida como una burocracia no politizada- aparece como un factor con una gran capacidad explicativa.

Por motivos de espacio, este resumen ha simplemente esbozado muchos de los argumentos y debates más relevantes en la literatura. Por ello, adjuntamos a continuación una lista con las referencias mencionadas en el texto, que representan los estudios más destacados de la última década en estos temas.

\section{BIBLIOGRAFÍA}

Acemoglu, Daron, Simon Johnson, and James A. Robinson. 2004. Institutions as the Fundamental Cause of Long-Run Growth.

Acemoglu, D., D. North, D. Rodrik, and F. Fukuyama. 2008. "Governance, Growth, and Development Decision-Making." World Bank.

Alt, James and David Lassen, The political economy of institutions and corruption in American States, Journal of Theoretical Politics 15 (3) (2003), pp. 341-365

Alt, James and David Lassen. Political and Judicial Checks on Corruption: Evidence from American State Governments. Economics \& Politics, Vol. 20, Issue 1, pp. 33-61.

Andrews, Josephine T., and Gabriella R Montinola. 2004. Veto Players and the Rule of Law in Emerging Democracies. Comparative Political Studies 37 (1):55-87.

Bäck Hannah, Axel Hadenius. 2008. "Democracy and State Capacity: Exploring a JShaped Relationship." Governance 21 (1): 1-24

Chang, Eric and Miriam Golden. 2006. "Electoral Systems, District Magnitude and Corruption”, British Journal of Political Science 37: 115-137.

Charron, Nicholas and Victor Lapuente. 2010.Does Democracy Produce Quality of Government?. European Journal of Political Research, (49) 443-470.

Charron, Nicholas, Carl Dahlström and Victor Lapuente. 2010. No Law without a State. QoG Working Paper Series 2010:12.

Charron, N., V. Lapuente and B. Rothstein (eds.).2010.Measuring the Quality of Government in the EU and Sub-national Variation, Report for the European Commission Directorate-General Regional Policy and Directorate Policy Development, EU 2010, Brussels.

Charron, Nicholas, Lewis Dijkstra and Victor Lapuente. 2010. "Bringing the Regions Back In. A study of quality of government across 172 EU regions", Annual Meeting of the American Political Science Association, Washington.

Clague, Christopher, Philip Keefer, Stephen Knack, and Mancur Olson. 1996. Property and Contract Rights in Autocracies and Democracies. Journal of Economic Growth 1 (2):243-76.

Dahlström, Calr, Victor Lapuente and Jan Teorell. 2009. "Bureaucracy, Politics and Corruption The Quality of Government Institute Working Papers Series, 2009/21.

Damania R, Fredriksson PG, List JA. 2003. Trade liberalization, corruption and environmental policy formation: theory and evidence. J. Envir. Econ. Manage. 46:490-512. 
Doig, Alan, and Stephen Riley. 1998. Corruption and Anti-Corruption Strategies: Issues and Case Issues and Case Studies from Developing Countries. In Corruption and Integrity Improvement Initiatives in Developing Countries, edited by in G. Shabbir Cheema, and Jean Bonvin, 45-62. Paris: OECD.

Dollar, D., R. Fisman and R. Gatti (2001), "Are Women Really the 'Fairer' Sex? Corruption and Women in Government", Journal of Economic Behavior and Organization, Vol. 46 (4): 423-429.

Easterly, William. 2001. The Elusive Quest for Growth: Economists' Adventures and Misadventures in the Tropics. Cambridge, Mass.: The MIT Press.

Erlingsson, Gissur Ó, Andreas Bergh och Mats Sjölin. 2008. "Public Corruption in Swedish Municipalities: Trouble Looming on the Horizon?”, Local Government Studies.

Estefanía, Joaquín (ed.). 2009. Report on Democracy in Spain/2009, Fundación Alternativas, Madrid.

Evans, Peter and James Rauch. 1999. "Bureaucracy and growth: A cross-national analysis of the effects of 'Weberian' state structures on economic growth". American Sociological Review 64 (4): 748-65.

Frey, Bruno S., and A. Stutzer. 2000. "Happiness, Economy and Institutions." The Economic Journal 110(466): 918-938.

Gupta S, Davoodi HR, Alonso-Terme R. 1998. Does corruption affect income inequality and poverty:Work. Pap. No. 98/76 (May), Int. Monetary Fund,Washington, DC.

Gupta S, Davoodi H, Tiongson E. 2000. Corruption and the provision of health care and education services.Work. Pap., Int. Monetary Fund,Washington, DC.

Hall, Robert E., and Charles I. Jones. 1999. Why Do Some Countries Produce So Much More Output Per Worker Than Others? Quarterly Journal of Economics 114 (1): 83116.

Harris-White, B. and G. White. 1996., Liberalization and New Forms of Corruption. Brighton: Institute of Development Studies.

Holmberg, Sören, Bo Rothstein, and Naghmeh Nasiritousi. 2009. “Quality of Government: What You Get”. Annual Review of Political Science Vol. 12: 135-161.

Johnston, Michael. 2005. Syndromes of Corruption: Wealth, Power, and Democracy. Cambridge: Cambridge University Press.

Kaufmann, Daniel, Aart Kraay and Pablo Zoido-Lobatón (1999a). “Aggregating Governance Indicators.” World Bank Policy Research Working Paper No. 2195, Washington, D.C.

Kaufmann, Daniel, Aart Kraay and Massimo Mastruzzi (2009). "Governance Matters VIII: Aggregate and Individual Governance Indicators for 1996-2008”. World Bank.

Policy Research Working Paper No. 4978. Washington, D.C.

Keefer, Philip. 2007. "Clientelism, Credibility, and the Policy Choices of Young Democracies." American Journal of Political Science 51 (4), 804-821.

Keefer, Philip, and Razvan Vlaicu. 2007. "Democracy, Credibility, and Clientelism.” Journal of Law, Economics, and Organization.

Knack, Stephen, and Philip Keefer. 1995. Institutions and Economic Performance: Cross-Country Tests Using Alternative Institutional Measures. Economics and PO-

$128 \quad \operatorname{litics} 7(3): 207-27$ 
Kurtz, M.J. and A. Schrank 2007. Growth and Governance: Models, Measures, and Mechanisms. Journal of Politics. Vol. 69, (2):

La Porta, Rafael, Florencio Lopez-de-Silanes and Andrei Shleifer. 2008. "The Economic Consequences of legal Origins". Journal of Economic Literature 46: 285-332.

La Porta, Rafael, Florencio Lopez-de-Silanes, Andrei Shleifer, and Robert Vishny. 1997. “Legal Determinants of External Finance". Journal of Finance, 52: 1131-1150.

La Porta, Rafael, Florencio Lopez-de-Silanes, Andrei Shleifer, and Robert Vishny. 1998. "Law and Finance". Journal of Political Economy, 106: 1113-1155.

La Porta, Rafael, Florencio Lopez-de-Silanes, Andrei Shleifer, and Robert Vishny. 1999. “The Quality of Government". Journal of Law, Economics, and Organization, 15: 222-279.

Lemarchand, Rene. 1972. "Political Clientelism and Ethnicity in Tropical Africa: Competing Solidarities in Nation-Building," American Political Science Review, 66, 1, 68-85.

Levchenko, A. 2007. "Institutional Quality and International Trade". Review of Economic Studies 74, 791-819.

Longstreth, M. and Shields, T. 2009. "A Comparison of Within Household Random Selection Methods for Random Digit Dial Surveys" Paper presented at the annual meeting of the American Association For Public Opinion Association, Fontainebleau Resort, Miami Beach, FL.

Mauro P. 1995. Corruption and growth. Q. J. Econ. 110:681-712.

Miller, Gary J. and Thomas Hammond. 1994. "Why Politics is more fundamental than Economics: Incentive-Compatible Mechanisms are not credible". Journal of Theoretical Politics 6 (1): 5-26.

Miller, Gary J. and Dino Falaschetti. 2001. "Constraining Leviathan: Moral Hazard and Credible Commitment in Institutional Design." Journal of Theoretical Politics, 13: 389-411.

Mo, P. H. 2001. "Corruption and Economic Growth." Journal of Comparative Economics 29: 66-79.

Morse, S. 2006. "Is corruption bad for environmental sustainability? A cross-national analysis." Ecology and Society 11(1): 22.

Near, J. P., and Miceli, M. P. (1985). "Organizational dissidence: The case of whistleblowing", Journal of Business Ethics, 4, 1-16.

Olsen, Johan P. 2005. "Maybe It Is Time to Rediscover Bureaucracy". Journal of Public Administration Research and Theory 16: 1-24.

Olsen, Johan P. 2008. "The Ups and Downs of Bureaucratic Organization". Annual Review of Political Science 11: 13-37.

Persson, Anna, Bo Rothstein and Jan Teorell. 2010. "The failure of Anti-Corruption Policies. A Theoretical Mischaracterization of the Problem", The Quality of Government Institute, University of Gothenburg, Working paper 2010: 19.

Persson, Torsten, Gerard Roland, and Guido Tabellini. 2000. "Comparative Politics and Public Finance." Journal of Political Economy, 108: 1121-1141.

Persson, Torsten, Guido Tabellini, and Francesco Trebbi. 2003. "Electoral rules and corruption", Journal of the European Economic Association 1(4): 958-989.

Putnam, Robert. 1993. Making Democracy Work. Princeton: Princeton University Press. 
Rauch, James and Peter Evans. 2000. "Bureaucratic structure and bureaucratic performance in less developed countries". Journal of Public Economics 75 (2000): 49-71.

Rodrik D, Subramanian A, Trebbi F. 2004. "Institutions rule: the primacy of institutions over geography and integration in economic development." Journal of Economic Growth 9(2): 131-165.

Rothstein, Bo. 2007. "Anti-Corruption - A Big Bang Theory”. The Quality of Government Institute, University of Gothenburg, Working paper 2007: 3.

Rothstein, Bo. 2009. Preventing Markets from Self-Destruction: The Quality of Government Factor The Quality of Government Institute, University of Gothenburg, Working paper 2009: 2.

Rothstein, Bo. 2010. "Corruption, Happiness, Social Trust and the Welfare State: A Causal Mechanisms Approach", The Quality of Government Institute, University of Gothenburg, Working paper 2010: 9.

Rothstein Bo and D. Eek. 2009. Political corruption and social trust-an experimental approach. Rationality \& Society 21:81-112.

Rothstein, Bo, and Dietlind Stolle. 2008. „The State and Social Capital: An Institutional Theory of Generalized Trust." Comparative Politics 40: 441-467.

Rothstein, Bo and Teorell, Jan. 2008. "What Is Quality of Government? A Theory of Impartial Government Institutions." Governance: An International Journal of Policy, Administration and Institutions 21(2): 165-190.

Scott, James C. 1972. Comparative Political Corruption, Englewood Cliffs, NJ: PrenticeHall.

Sung, Hung-En. 2003. "Fairer Sex or Fairer System? Gender and Corruption Revisited”, Social Forces, Vol. 82 (2): 703-723.

Sung, Hung-En. 2004. "Democracy and Political Corruption: A Cross-National Comparison", Crime, Law and Social Change, Vol. 41 (2): 179-193.

Tabellini, Guido, 2008, "Institutions and Culture", The Journal of the European Economic Association, Presidential Lecture to the European Economic Association, April.

Tabellini, Guido, 2009, "Culture and Institutions: Economic Development in the Regions of Europe", The Journal of the European Economic Association, forthcoming.

Transparency International. 2006. Global Corruption Report 2006. London: Pluto.

Treisman, Daniel. 2000. "The Causes of Corruption: A Cross-National Study", Journal of Public Economics, Vol. 76: 399-457.

Treisman, Daniel. 2007. "What Have We Learned About the Causes of Corruption from Ten Years of Cross-National Empirical Research?”, Annual Review of Political Science 10: 211-44.

Uslaner, Eric M. 2008. The Bulging Pocket and the Rule of Law. Corruption, Inequality and Trust. New York: Cambridge University Press.

Wade, Robert. 1985. "The Market for Public Office: Why the Indian State is not Better at Development." World Development, 13, 467-97.

Weber, Max. 1978. Economy and Society. Roth, Guenther and Claus Wittich. eds. Berkeley: University of California Press.

Welsch H. 2004. Corruption, growth, and the environment: a cross-country analysis.

130 Environ. Dev. Econ. 9:663-93 
Welzel, Christian and Ronald Inglehart. 2008. "The Role of Ordinary People in Democratization." Journal of Democracy, 19 (1): 126-140.

World Bank. 1997. World. Development Report 1997: The State in a. Changing World New York: Oxford University.

Wängnerud, Lena. 2009. "Women in Parliaments. Descriptive and Substantive Representation." Annual Review of Political Science Vol. 12: 51-69.

Öberg, Magnus and Melander, Erik. 2010. Autocracy, Bureaucracy, and Civil War. APSA 2010 Annual Meeting Paper. Available at SSRN: http://ssrn.com/abstract=1642997.

Recibido: XX de mes de año

Aceptado: $X X$ de mes de año 
